\title{
Entretien avec Michelle Perrot
}

Itinéraire d'une pionnière

Florence Rochefort et Françoise Thébaud

\section{(2) OpenEdition \\ Journals}

Édition électronique

URL : https://journals.openedition.org/clio/9909

DOI : 10.4000/clio.9909

ISSN : 1777-5299

Éditeur

Belin

Édition imprimée

Date de publication : 31 décembre 2010

Pagination : 217-231

ISBN : 978-2-8107-0098-1

ISSN : 1252-7017

Référence électronique

Florence Rochefort et Françoise Thébaud, « Entretien avec Michelle Perrot », Clio. Femmes, Genre, Histoire [En ligne], 32 | 2010, mis en ligne le 31 décembre 2012, consulté le 28 avril 2022. URL : http:// journals.openedition.org/clio/9909; DOI : https://doi.org/10.4000/clio.9909 


\section{Entretien avec Michelle Perrot. Itinéraire d'une pionnière}

\section{Les conditions de production de ce texte sont précisées}

ci-dessous par Michelle Perrot.

L'équipe de Clio HFS m'a demandé de participer au numéro «Relectures» préparé pour le 15e anniversaire de la revue. Nous devions le faire sous forme d'interview que nos indisponibilités réciproques ont rendu impossible. Je réponds donc, par écrit, à quelques-unes des questions posées par Florence Rochefort et Françoise Thébaud. L'inconvénient est la perte de spontanéité que suscite l'inattendu d'un entretien. J'ai plutôt le sentiment de m'inscrire en l'occurrence dans la suite des Essais d'ego-histoire, lesquels, réalisés au milieu des années 1980, s'arrêtaient justement au seuil de l'histoire des femmes, devenue pourtant, et déjà depuis une dizaine d'années, ma préoccupation majeure. Il faudrait beaucoup plus de temps et de réflexion pour une évocation de ce second volet d'une recherche où l'individuel se confond avec le collectif, qui lui suggère, voire qui lui dicte, ses interrogations et influence ses démarches. Que dois-je, une fois de plus, à «l'air du temps » ${ }^{1}$ dont j'ai sans douté été excessivement tributaire? Quelle a été ma part d'initiative dans la mise au jour, ou le renouvellement, de ces problématiques? De quoi ai-je été sinon l'inspiratrice, du moins la traductrice ? Comment éviter l'aspect narcissique, ou téléologique, de telles rétrospectives? Le hasard et l'injustice des choix mémoriels ? Sur les chemins du petit Poucet, voici quelques cailloux.

Florence Rochefort et Françoise Thébaud : Quelles ont été, dans votre parcours d'historienne des femmes, les lectures, les rencontres, les collaborations (françaises ou étrangères) les plus importantes?

1 Titre de ma contribution aux Essais d'ego-histoire: Perrot 1987. 
Parmi les noms qui nous viennent à l'esprit : Michel Foucault, Arlette Farge, Alain Corbin, Geneviève Fraisse, Mona Ozouf, Robert Badinter, Georges Duby, Joan Scott...

Michelle Perrot : Oui, tous ceux-là et bien d'autres. Tant de noms me viennent à l'esprit que la sélection est presque impossible et nécessairement arbitraire. J'évoquerai plutôt des strates, des groupes. Le trio que, jeunes professeurs débutantes, nous formions, au milieu des années 1950, au lycée de jeunes filles de Caen - Mona Ozouf (philosophie), Nicole Le Douarin (sciences naturelles) et moi (histoire) - a eu une influence majeure pour nos choix, professionnels et autres. Mona et moi avons eu récemment l'opportunité d'évoquer cette période et cette longue amitié dans les "Mélanges" offerts à Nicole, la biologiste, mondialement connue, nobélisable, qui a inventé les «chimères » et a contribué à la découverte de l'ADN et des cellules-souches ${ }^{2}$. Aucune d'entre nous n'était, il est vrai, engagée dans les recherches sur les femmes; dans cette France de la Reconstruction, dominée par l'économique et le social, elles n'étaient pas notre souci premier; mais nous nous sentions très concernées par la question des relations entre les sexes. Brigitte Bardot (Et Dieu créa la femme), Simone de Beauvoir, que nous admirions beaucoup plus que nos compagnons, ou Jeannette Vermeersch, qui choquait notre féminisme latent ${ }^{3}$, suscitaient dans notre groupe des discussions passionnées. Dans leurs livres ultérieurs, Mona (Les mots des femmes, Composition française) et Nicole (divers papiers à l'Académie des sciences et autobiographie en cours) ont eu l'occasion de revenir sur la question des femmes à propos du travail, de la famille, ou de la transmission, dans leur propre parcours.

La deuxième strate est celle du Mouvement Social, fondé en 1961 par Jean Maitron ${ }^{4}$ : Madeleine Rebérioux et Rolande Trempé, qui se sont

2 Ozouf \& Perrot 2010.

3 Annette Wieviorka consacre un chapitre aux « vices de la bourgeoisie», pour reprendre l'expression de Jeannette relative aux pratiques contraceptives: Wieviorka 2010.

4 Assistante de Labrousse à la Sorbonne, coorganisatrice du colloque sur «Le militant ouvrier », je faisais partie de l'équipe fondatrice. 
impliquées dans l'histoire des femmes. La première à Vincennes, la seconde, surtout, à Toulouse, avec Marie-France Brive et dans ses recherches sur les femmes dans la Résistance (cf. son film à cet égard). A travers elles, on mesure ce que l'histoire des femmes doit à l'histoire sociale, et les difficultés qu'elle éprouve à s'en dégager. Madeleine nous a quittés; Rolande me demeure très proche.

Je voudrais dire aussi ce que je dois aux historiennes anglaises (Siân France Reynolds, passée de l'histoire ouvrière à celle des femmes ${ }^{5}$ ) et américaines, Natalie Zemon Davis, Joan Wallach Scott et Louise Tilly, toutes trois spécialistes de la France. Natalie, pionnière de l'histoire culturelle et des Women's studies, m'avait accueillie à Berkeley, puis à Princeton ; j'avais été éblouie par son intelligence, sa beauté et par une originalité qui n'a cessé de s'affirmer: "Women on top ", Trois femmes en marge au XVII e siècle, sont des textes majeurs. Joan et Louise étaient au départ des historiennes du travail, très soucieuses de la dimension du genre ${ }^{6}$, dont Joan allait devenir la principale théoricienne. En 1978, notre trio avait proposé un thème sur "Femmes et industrialisation", dans le cadre des tables rondes organisées, dans les années 1975-1980, à la Maison des sciences de l'homme à Paris. Clemens Heller, directeur de la MSH, appuyé par Georges Haupt, souhaitait créer un réseau européen d'histoire sociale, alors au zénith; on y retrouvait, outre des français (tels que Yves Lequin et Patrick Fridenson, Madeleine, Rolande et moi), David Montgomery, Charles Tilly, Eric Hobsbawm, E.P. et Dorothy Thompson. Si Dorothy était notre complice, les hommes étaient plus réservés, un peu inquiets de voir le sexe déborder la classe. Ce fut l'occasion de discussions assez vives, riches de prolongements.

En ce qui concerne l'histoire des femmes proprement dite, les rencontres des années 1970 ont été décisives, notamment à Paris 7 Jussieu et à l'EHEss. A Paris 7, Françoise Basch, Françoise Barret-

5 Ce passage est très sensible dans sa thèse sur Jean Allemane (soutenue à Paris sous ma direction), par le souci de faire sa place à la vie privée, aux relations avec les femmes.

6 Scott \& Tilly 1978. On sait qu'elles ont divergé par la suite, Joan devenant la principale théoricienne du gender, Louise, plus amarrée au social, demeurant réticente. 
Ducrocq, puis Rita Thalman et Marie-Claire Hoock-Demarle ont élargi considérablement mon horizon européen et international. En 1973, avec Pauline Schmitt et Fabienne Bock, nous lancions le premier cours intitulé «Les femmes ont-elles une histoire?»", prélude à beaucoup d'autres. Ce secteur nouveau de recherches attirait les étudiantes et quelques étudiants. Maitrises et thèses se multiplièrent, créant un véritable champ que Françoise Thébaud a décrit. Les années 1970-1980 furent une période d'intense fermentation, qui correspond à la grande période du mouvement de libération des femmes. Dans cette dynamique, il est difficile d'individualiser son propre parcours. En ce qui me concerne, j'ai eu le sentiment, plus qu'ailleurs, de dissiper des silences, de retrouver les femmes (y compris ma mère), et de faire coïncider mon identité et celle d'un « nous » refoulé.

Dans un séminaire informel de l'EHESS, dont Cécile Dauphin était une cheville ouvrière, nous tentions de nous approprier les savoirs anthropologiques (Maurice Godelier, les Américaines) et historiques (Georges Duby) sur les femmes. C'était un cercle de lecture très efficace. Nous nous retrouvions dans les premiers colloques, celui d'Aix en 1976 et celui de Saint-Maximin en 1984, avec Yvonne Knibiehler et ses collègues du CEFUP. C'est à l'EHESS que s'est formée l'équipe de rédaction de l'Histoire des femmes, autour de Pauline Schmitt, Christiane Klapisch-Zuber, Arlette Farge, Geneviève Fraisse, auxquelles s'est jointe Françoise Thébaud, la benjamine, auteure, avec Mathilde Dubesset et Catherine Vincent, de la toute première maitrise «femmes» (sur les munitionnettes des usines de la Première Guerre mondiale) soutenue à Paris VII. Ce fut aussi l'aventure de Pénélope, fruit d'une collaboration entre l'École et Jussieu; et l'écriture de l'article-manifeste des Annales: "Culture et pouvoir des femmes. Essai d'historiographie $»^{8}$.

Je voudrais dire aussi ce que je dois à l'équipe du Mage et d'abord à Margaret Maruani, que j'ai suivie dans toutes ses entreprises, à Janine Mossuz-Lavau, introductrice du genre à Sciences-Po; à Evelyne Pisier, que je connus au Conseil national des programmes,

\footnotetext{
7 Basch 2001.

8 Mars-avril 1986.
} 
où nous tentions d'introduire les femmes; à Françoise Héritier, qui fut ma présidente au Conseil national du Sida, fondé en 1989 par François Mitterrand, où nous retrouvions Daniel Defert, créateur de AIDES, en mémoire de son ami Michel Foucault, mort du sida en 1984. J'ai apprécié l'engagement de Françoise Héritier et la pertinence de sa réflexion d'anthropologue sur la maladie et la différence des sexes ${ }^{9}$. Sans partager nécessairement le point de vue structuraliste qui est le sien, j’ai incorporé son apport magistral sur Masculin/Féminin. La pensée de la différence ${ }^{10}$. J'ai eu plaisir à participer à son portrait, réalisé par Teri Damisch, dans la série "Empreintes », diffusée par France 5 (2008).

J'ai cité surtout des femmes, majoritaires dans un champ de recherches qu'elles ont créé et investi. Mais je venais de terres «masculines », qu'en vérité je ne voyais pas comme telles, réservée devant les perspectives de "rupture épistémologique » avancées par certaines de mes amies. Sans doute étais-je trop timide, et - déjà trop vieille. Parmi les historiens, qui, pour des raisons diverses, ont compté pour moi, je mentionnerai Ernest Labrousse, Georges Duby, Alain Corbin: trois générations, trois moments de mon parcours. Alain, c'est notre jeunesse. J'étais certes l'aînée. Il était étudiant à Caen quand je débutais au lycée Pasteur. Nous nous retrouvions autour de l'immense table ovale des archives départementales, où il faisait sa maitrise, tandis que je commençais mes recherches sur les grèves. Nous (Jean-Claude et moi) l'appelions « le beau ténébreux ». Il fit sa thèse sous la direction de Labrousse, mais il prit rapidement ses distances pour une dimension plus originale, l'histoire des «sensibilités », qu'il n'a cessé d'approfondir de la manière que l'on sait. Nous avons constamment collaboré, dans les séminaires, les jurys de thèses, et en particulier pour le tome IV de l'Histoire de la vie privée, initiée par Philippe Ariès et Georges Duby, que je dirigeai ; ce fut, entre 1982 et 1986, avec Roger-Henri Guerrand et Anne MartinFugier, un vrai travail d'équipe ; Alain a donné à ce volume des pages

9 J'ai eu l'occasion de le dire dans un texte des Mélanges qui lui ont été offerts : «La Présidente ».

10 Héritier 1996, 2002. Les discussions avec les historiennes ont peut-être influé sur la rédaction de ce second volume qui fait place à « l'évènement » contraceptif. 
éblouissantes : la $4^{\mathrm{e}}$ partie, "Coulisses », la plus suggestive. Participant de l'histoire des femmes par ses travaux propres (Les Filles de noce, 1978, est un des premiers ouvrages sur la prostitution), Alain Corbin fut un "compagnon de route » attentif et critique ${ }^{11}$. Pour ma part, je lui dois le meilleur compte-rendu de mon dernier livre, Histoire de chambres (Seuil, 2009), dans le cadre de La vie des idées (Ivan Jablonka) ${ }^{12}$. C'est un demi-siècle d'échanges, personnels et professionnels, qu'il faudrait ici décrire.

Labrousse et Duby, c'est autre chose. Ernest Labrousse, grand maître de l'histoire économique et sociale, fut le mien, et j'ai dit ailleurs tout ce que je lui dois. Résolument égalitaire dans ses pratiques, il n'était pas spécialement féministe; les questions de sexe lui étaient étrangères, et le « genre» n'aurait eu pour lui aucune signification. Son évocation du rôle des femmes dans les émeutes de subsistances de l'Ancien régime, dans un cours fameux de l'amphi Descartes, est une image qui m'avait marquée ${ }^{13}$.

Georges Duby fut l'initiateur de la « nouvelle histoire». Sa merveilleuse écriture y fit entrer les jeunes, les femmes, l'amour, la violence, la beauté : une esthétique dans un paysage un peu gris, une respiration dans un espace corseté. Dans Mâle Moyen Age, dans Le chevalier, la femme et le prêtre, une de nos lectures-clef au séminaire de l'École, et dans tant d'autres textes, il posait la question de l'invisibilité des femmes - «Les femmes : que sait-on d'elles ? - et de la domination masculine. Rétrospectivement, je suis frappée de la prégnance de cette idée au Collège de France, si l'on songe aux travaux de Duby, Bourdieu, Foucault et, plus tard, de Françoise Héritier. Avec Ariès, G. Duby fut le promoteur de la Vie privée $e^{14}$ et

11 Corbin 1984. Voir Perrot 2004.

12 Alain Corbin, "Toute l'intimité du monde», à l'occasion d'un débat fin septembre 2009 au Collège de France. www.laviedesidees.fr.

13 On en retrouve l'écho dans «La femme populaire rebelle », publié dans L'bistoire sans qualités (Dufrancatel 1979), un des premiers livres collectifs sur l'histoire des femmes (avec Arlette Farge, Geneviève Fraisse, Christine Fauré...), que nous avions tellement de mal à dénommer, tant nous redoutions de constituer un ghetto! Nos discussions à ce sujet furent épiques; article repris dans Perrot $1998: 153-176$.

145 volumes parus au Seuil, 1985-1987. 
c'est à ce sujet qu'il me sollicita. J'ai raconté ailleurs comment, dans la foulée, et à la demande de l'éditeur italien Laterza, il me proposa l'entreprise qui allait devenir l'Histoire des femmes ${ }^{15}$. Entreprise collective, considérable, où j'ai joué nécessairement le rôle de médiatrice, un peu trop sans doute, au gré des directrices de volume, qui regrettaient l'absence de contact direct avec un Georges Duby plus lointain. D'où des malentendus, à propos notamment de la publication d'Images de femmes ${ }^{16}$ et du colloque de la Sorbonne en 1992, Femmes et Histoire ${ }^{17}$. Peu de choses, à mon sens, au regard de l'apport de Georges Duby, auquel Clio HFS a rendu hommage ${ }^{18}$. Cette confluence entre lui et nous a contribué à faire entrer l'histoire des femmes dans l'Histoire. Peut-on dire que c'est au prix de certains renoncements ? Certaines historiennes américaines ont critiqué ce qui leur apparaissait au fond comme une « collaboration de sexes ».

Mes collaborations avec Michel Foucault et Robert Badinter sont d'un autre ordre, et dissemblables quoiqu'elles se situent dans une certaine continuité. Les mots me manquent pour dire ce que je dois à l'œuvre de Michel Foucault ${ }^{19}$, en particulier (mais pas seulement) à Surveiller et Punir, maitre-livre sur la naissance de la prison et plus largement sur la généalogie des disciplines. Dans sa mouvance, j’ai développé, avec Arlette Farge (entre 1976 et 1979, nous dispensions ensemble à Jussieu un cours sur ces questions), les recherches sur la criminalité et la pénalité, devenues un champ d'investigation très dynamique. Par rapport à l'histoire des femmes, on pourrait dire que c'était un contre-champ. D'une part, les femmes sont, pour des raisons complexes, relativement, et de façon croissante à l'époque contemporaine, absentes de cette sphère. Elles représentent aujourd'hui moins de $4 \%$ de la population carcérale. Du reste, leur moindre délinquance comme leur moindre punition, pose un

15 C'est à la suite d'un colloque à Genève, où Pauline Schmitt et moi présentions ce qui était encore en gestation, que, devant les objections d'un participant qui nous reprochait de prendre pour universel un modèle occidental, nous proposâmes d'ajouter « en Occident ».

16 Duby \& Perrot 1992.

17 Femmes et Histoire, 1993. Voir à cet égard : Farge 2005.

18 Klapisch-Zuber 1998.

19 Perrot 2001. 
véritable problème de différence des sexes. D'autre part, ce fut un secteur de recherches viril. Tandis que les étudiantes travaillaient sur les femmes, leurs camarades s'intéressaient au crime et au châtiment, reproduisant de manière presque caricaturale la sempiternelle division des sexes. C'est par le crime et la prison que j'ai connu Jacques-Guy Petit, Dominique Kalifa, Marc Renneville, Jean-Christophe Coffin, Philippe Artières, Eric Pierre, Jean-Claude Vimont, Jean-Jacques Yvorel et quelques autres. Bien entendu, j'essayais de croiser les deux, proposant des sujets sur l'infanticide, l'avortement, les émeutes frumentaires, les délits forestiers, le vol de grands magasins, les prisons de femmes ${ }^{20}$. Mais j'étais frappée de cette polarité. C'est peutêtre à ce propos que j'ai senti le plus vivement la nécessité d'une analyse en termes de genre ${ }^{21}$. Celle-ci n'était pas un souci majeur de Michel Foucault, même si on trouve dans la « boîte à outils » qu'il nous a laissée bien des instruments pour y procéder ${ }^{22}$.

C'était identique avec Robert Badinter. L'ancien Garde des Sceaux, devenu président du Conseil constitutionnel, souhaitait poursuivre la réflexion de Foucault sur la pénalité notamment pour la période contemporaine. Celui qui fut « notre meilleur ministre de la Justice » (dixit Foucault), supportait mal la critique que le philosophe faisait des Lumières et voulait comprendre les raisons de la défaillance démocratique en matière carcérale. Il me demanda d'organiser un séminaire qui, durant cinq ans (1986-1991), réunit à l'EHESS historiens, juristes, médecins, personnel pénitentiaire, autour de «la prison républicaine », titre de l'ouvrage qui en est sorti23. Ce travail commun était exigeant et riche d'implications et de rencontres (celle de Mireille Delmas-Marty par exemple, de Béatrice Koeppel et de Catherine Prade, conservatrice du musée pénitentiaire de Fontainebleau). Il n'était pas toujours aisé à croiser avec l'histoire des femmes où, pourtant, la dimension du droit était fondamentale.

20 Cf. la thèse pionnière de Claudie Lesselier sur les prisons de femmes au XIX siècle : Lesselier 1982 ; et les travaux de Martine Kaluzynski, de Françoise Tétard $(† 2010)$.

21 Depuis quelques années, les recherches sur la délinquance féminine se sont multipliées. Dernier exemple en date : Chauvaud \& Malandon 2009.

22 Michelle Perrot, " Michel Foucault et l'histoire des femmes », in Perrot 2001.

23 Badinter 1992. 
Pendant une dizaine d'années, j'ai eu l'impression de vivre dans deux domaines disciplinaires sexuellement différents. Ce qui me posait beaucoup de questions intellectuelles et personnelles.

FR et FT : Quel lien faites-vous ou que signifie pour vous le passage de l'histoire de la vie privée à l'histoire des femmes ? Utilisez-vous, et si oui pourquoi, le terme d'histoire du genre?

MP : En vérité, ces deux «histoires» se sont développées parallèlement plus que successivement. Si on regarde la chronologie, il y a même antériorité de l'histoire des femmes (1973-1980). C'est sans doute à cause d'elle que Georges Duby, informé de ces recherches, m'a (en 1982) « requise » pour la vie privée, établissant en somme entre privé et femmes une équivalence assez classique, mais qui ne manque pas d'ambiguité. Chargée du tome 4 (le XIXe siècle, qu'on peut considérer comme un âge d'or de la vie privée), j'organisai à Jussieu (pour moi un véritable laboratoire) cours et séminaires à ce sujet. J'y retrouvai les femmes, bien entendu, dans la famille, la maison, l'intimité, les travaux et les jours, mais d'une manière plus problématisée qui menait sur les chemins du genre. Ce détournement de l'histoire des femmes lui a sans doute, au bout du compte, bénéficié.

Cette histoire, nous l'avons conçue très tôt comme relationnelle (cf. colloque d'Aix en 1976). Nous l'avions développée grosso modo selon deux versants principaux : les femmes victimes; la culture des femmes. L'un et l'autre posaient la question de la domination masculine et des systèmes de représentation. «Cette histoire se veut celle du rapport des sexes plus que des femmes. C'est cela sans doute le nœud du problème, et qui définit l'altérité et l'identité féminines ", écrivions-nous dans l'Introduction de l'Histoire des femmes. Pourtant, nous employions peu le mot " genre » et généralement sous sa forme anglaise. Ainsi Pauline Schmitt parle, dans son introduction au tome 1 (Antiquitê) des "discours masculins sur la différence des sexes, le "genre" (gender) ${ }^{24}$. Je pourrais citer d'autres occurrences antérieures. Françoise Thébaud a fait ce travail et montré la lente pénétration du

24 Schmitt Pantel $2001: 25$. 
«genre» en France. À la fois pour des raisons de vocabulaire (les ambiguïtés du « genre » en langue française) et d'épistémologie. La prégnance de l'histoire sociale a été un obstacle à la pénétration du linguistic turn et un positivisme persistant a freiné la prise en compte du symbolique. Je n'apporterai pas grand chose à une historiographie désormais largement abordée. Je rapporterai seulement une anecdote rétrospectivement significative.

J'avais, pour le tome 4 de l'Histoire des femmes, sollicitée (en 1989) Joan Scott afin de traiter des ouvrières, qu'elle connaissait si bien. Je pensais (candidement sans doute) à une analyse assez classique d'histoire sociale. Elle accepta, tout en me disant qu'elle ne prendrait pas les choses de cette manière trop descriptive, mais sous l'angle plus méthodologique des discours qui constituent la travailleuse en objet: discours de l'économie politique, du patronat et des syndicats qui justifient la division sexuelle du travail et la séparation du lieu de travail et du domicile en les naturalisant. «La division sexuelle du travail : produit de l'histoire, effet de discours $»^{25}$, telle était son optique. Brillante illustration de la théorie du Genre «catégorie utile de l'analyse historique ", selon le titre de son article fameux de $1986^{26}$. Je n'avais pas anticipé une telle réponse qui me surprit, alors même que j'avais lu, mais incomplètement assimilé, son article; et c'est caractéristique d'un certain retard conceptuel.

Le terme s'est depuis acclimaté et généralisé, au point de susciter récemment des mises en garde officielles. Il a contribué à déplacer la focale (des femmes à «la différence des sexes »), à problématiser et élargir les perspectives, en englobant l'histoire de la masculinité, celle des homosexualités, de la transsexualité. Les Gender studies, auxquelles j'ai adhéré, sans vraiment y contribuer, me paraissent aussi plus faciles à implanter dans les structures académiques. Il s'en crée à Sciences Po, dans nombre d'universités de province ou des Dom (ainsi à la Martinique, à la Réunion). Le Genre est un outil d'analyse déjà critiqué et partiellement dépassé. Mais il n’a pas épuisé ses effets.

Je me sens ici témoin plus qu'actrice.

\footnotetext{
25 Scott 1991.

26 Connu surtout par sa reprise dans Scott 1988.
} 
FR et FT : Il nous semble que vous avez assumé et assumez avec brio une fonction de passeuse. Pouvez-vous nous en dire plus?

MP : J'ai eu la chance de vivre une phase sinon unique, du moins privilégiée, de la vie universitaire. On n'imagine plus la vitalité des nouvelles universités fondées après 1968, l'atmosphère de liberté antihiérarchique qui y régnait, même si les conflits étaient fréquents et le travail considérable en raison de la diversité des tâches à assumer. Docteure en 1971, professeure en 1974, j'en ai largement profité. Jussieu a été pour moi un laboratoire, où recherche et enseignement étaient liés (nous devions enseigner à tous les niveaux). La direction de maitrises et de thèses, autour de séminaires ouverts et fréquentés (je les concevais comme des rendez-vous hebdomadaires où venait qui voulait), a été un moyen particulièrement efficace de construction des deux domaines que j'avais choisi de développer: histoire des femmes, histoire de la pénalité. Les thèses sont un vrai contact avec la recherche vivante. Nombre des docteur(e)s et des étudiant(e)s que j’ai suivis (dans tous les sens du mot) ont pris la relève au CNRS, ou à l'université et occupent aujourd'hui des postes de responsabilité. Non sans difficultés, parce que l'histoire des femmes, populaire dans le public, n'a jamais été totalement acceptée et reconnue dans le monde académique. Il faut une certaine abnégation pour la pratiquer. C'est une limite qui me pose problème : qu'avons-nous manqué ?

Bien entendu, je ne dirige plus de thèses et je ne fais plus partie de jurys (à quoi bon lorsqu'on est en surnombre? C'est du temps perdu). Mais j'ai accordé et j'accorde du temps aux médias. J'ai longtemps été collaboratrice du jeudi des « livres» dans Libération, où œuvrent actuellement Yannick Ripa et Dominique Kalifa, deux anciens de Jussieu. J'ai présidé pendant trois ans les «Rendez-vous de l'Histoire de Blois ", dont une session, fort réussie, a été consacrée aux « Femmes dans l'Histoire ». Je fais partie de l'équipe productrice des «Lundis de l'Histoire» et m'efforce d'y maintenir la thématique «Femmes/Genre ». En juin 2010, autour de "Amitiés et amours», j’ai reçu Anne Vincent-Buffault (Histoire de l'amitie) et Julian Jackson (Arcadie: une des premières associations gays d'après-guerre); en août, on entendra Annette Wieviorka pour Maurice et Jeannette. Biographie du couple Thorez. (Fayard): un livre passionnant; en 
septembre, je recevrai Christine Bard pour son Histoire politique du pantalon (Seuil); en octobre, ce sera la folie avec Yannick Ripa (l'étonnante affaire Hersilie Rouy, Tallandier) et Laurence Guignard (Juger la folie, PUF). C'est une position privilégiée pour être au courant des parutions et les faire connaitre ; je regrette toutefois que l'heure dont nous disposons (le lundi après-midi de 15h à 16h; au lieu de $10 \mathrm{~h}$ le matin, auparavant) soit si peu favorable. J'ai eu également l'occasion de consacrer sur France Culture une série d'émissions à l'histoire des femmes, publiées sous le titre un peu ironique de Mon histoire des femmes ${ }^{27}$. J'aime beaucoup la radio (pas la télévision, parce que je déteste mon image), et j'en aurais volontiers fait mon métier.

J'aime moins rédiger des préfaces, bien qu'on m'en demande souvent. Cela prend beaucoup de temps et, à mon avis, cela ne sert à rien pour le livre qu'elles sont censées défendre. Malgré tout, c'est un moyen de dire sa solidarité avec tel texte ou telle manifestation. A pratiquer avec modération.

FR et FT: Vos travaux d'aujourd'hui et de demain : les chambres, George Sand... Pouvez-vous nous en parler?

MP : Histoire de chambres ${ }^{28}$ est sans doute mon livre le plus personnel. J'ai eu beaucoup de plaisir à le concevoir et à l'écrire. Certains y voient un aboutissement (Alain Corbin par exemple); d'autres une aventure inattendue, voire une rupture. Je ne sais. En tout cas, c'est un essai où, loin de tout académisme, je me suis donné beaucoup de liberté. Du moins l'aurais-je souhaité, et bien davantage. J'avais même pensé à le publier sous un pseudonyme: complexe d'Emile Ajar? "On vous aurait reconnue », me dit un collègue et ami. Il est bien difficile de changer d'identité, plus encore de repousser ses limites, des limites que j'ai constamment senties, dans la pensée comme dans l'écriture. Il faut se résigner à n'être que soi.

Le livre a reçu le prix Femina Essai (2009) et a eu un succès que je n'attendais pas, comme si les gens découvraient l'évidence de la

$\begin{array}{ll}27 & \text { Perrot } 2006 . \\ 28 & \text { Perrot } 2009 .\end{array}$ 
chambre devenue sujet ${ }^{29}$. J'ai reçu beaucoup de courrier, critique, amusant, touchant. J'ai couru la France dans d'innombrables librairies, sociétés, salons du livre. Sans doute ne retrouverai-je jamais ce contact avec le public.

George Sand, que j’avais longtemps dédaignée, m'est devenue familière ${ }^{30}$ depuis une quinzaine d'années. En publiant ses textes politiques (1843-1850), son Journal d'un voyageur pendant la guerre, j'ai modestement contribué à la sortir de son image de "bonne dame ", qui m'agaçait dans ma jeunesse. Le renouveau des études sandiennes, en partie grâce au féminisme, français et américain, m’a permis de rencontrer un milieu de littéraires (Nicole Mozet, Christine Planté, Martine Reid, José-Luis Diaz...) et d'amateurs très attachant, où je suis quasi la seule historienne (excepté Bernard Hamon ${ }^{31}$ ). Nouvelles rencontres, nouvelles amitiés; plongée dans la littérature que je n'ai cessé de retrouver, de revaloriser. Elle est aujourd'hui mon horizon préféré bien loin des trois tomes du Salaire, l'évolution sociale et la monnaie, de François Simiand, dont Ernest Labrousse me disait qu'ils devaient être mon livre de chevet. J'ai le projet de faire un livre sur Nohant, village et maison d'Aurore/Sand, haut-lieu du romantisme dans un Berry déconcertant, méconnu, «le pays du sommeil et de la mort ", disait George les jours de spleen ; de le prendre dans toutes ses dimensions, économiques, sociales (domesticité), familiales, amicales, ethnographiques, culturelles, artistiques (la musique, le théâtre), politiques, sans oublier le jardin. Je ne connais pas d'autre maison d'écrivain, d'autre maison de femme, qui ait cette densité de sociabilité, de création et d'intrigues. Je voudrais prendre le lieu dans sa totalité spatiale et relationnelle. L'historiographie est foisonnante (trop). Les sources sont très riches, notamment une prodigieuse correspondance : les vingt-six volumes publiés par Georges Lubin constituent un témoignage unique sur le XIXe siècle; mais aussi les

29 Cf. la livraison de La Quinzaine Littéraire, "Les chambres », été 2010 : sous la direction de Tiphaine Samoyault et Hugo Pradelle, réunit un florilège de textes d'auteurs actuels.

30 Je devrais dire « familier ». Sand parlait toujours d'elle comme auteur au masculin. Elle était « Sand» à Paris, Aurore au village.

31 B. Hamon a renouvelé la connaissance de Sand sous l'angle politique et religieux. 
Agendas, une œuvre impressionnante, romanesque et théâtrale, volumineuse, des paysages rêveurs.

C'est attirant. Un luxe sans doute, dont je ne voudrais pas malgré tout qu'il soit un « ouvrage de dames ».

\section{Bibliographie}

BADINTER Robert, 1992, La prison républicaine, Paris, Fayard.

BASCH Françoise et al. (dir.), 2001, Vingt-cinq ans d'etudes féministes. L'expérience Jussieu, Paris, Publications universitaires Denis-Diderot-Paris 7.

Chauvaud Frédéric \& Gilles Malandon (dir.), 2009, Impossibles victimes, impossibles coupables. Les femmes devant la justice (XIXe-XXe siècles), Rennes, Presses Universitaires de Rennes.

CORBIN Alain, 1984, "Le "sexe en deuil" et l'histoire des femmes au XIXe siècle », in Michelle Perrot (dir.), Une histoire des femmes est-elle possible ?, Marseille, Rivages, p. $142-154$.

DAVIS Natalie Zemon, Juive, catholique, protestante : trois femmes en marge au XVII siècle, Paris, Seuil.

Duby Georges \& Michelle Perrot, 1992, Images de femmes, Paris, Plon.

Dufrancatel Christiane et al., 1979, L'bistoire sans qualités, Paris, Éditions Galilée.

FARGE Arlette, 2005, «Et les femmes dans tout cela? », in Quel bruit ferons-nous? Entretiens avec Jean-Christophe Marti, Paris, Les Prairies ordinaires, p. 95-102.

Femmes et Histoire, 1993, colloque organisé par Georges Duby, Michelle Perrot et les directrices de L'bistoire des femmes en Occident, La Sorbonne, 13-14 novembre 1992, Paris, Plon.

Héritier Françoise, 1996, Masculin/Féminin, I, La pensée de la différence, Paris, Odile Jacob.

—, 2002, Masculin/Féminin, II, Dissoudre la biérarchie, Paris, Odile Jacob.

KLAPISCH-Zuber Christiane (dir.), 1998, " Georges Duby et l'Histoire des femmes », Clio. Histoire, Femmes et Sociétés, 8.

LESSELIER Claudie, 1982, «Les femmes et les prisons, 1815-1939 », thèse d'histoire, Université de Paris 7.

Ozouf Mona, 1995, Les mots des femmes, Paris, Fayard.

_, 2009, Composition française. Retour sur une enfance bretonne, Paris, Gallimard. 
Ozouf Mona \& Michelle Perrot, 2010, "Princesse de science", in Catherine Puigelier (dir.), Mots de science. Mélanges en l'bonneur de Nicole M. Le Douarin, Bruxelles, Bruylant, p. 155-171.

PERrot Michelle, 1987, «L'air du temps », in Pierre NORA (dir.), Essais d'ego-histoire, Paris, Gallimard, p. 241-292.

—, 1998, Les femmes ou les silences de l'histoire, Paris, Flammarion (coll. "Champs», 2001).

—, 2001, Les ombres de l'bistoire. Crime et châtiment au XIX'e siècle, Paris, Flammarion (coll. «Champs », 2002).

—, 2004, «Alain Corbin et l'histoire des femmes », French Politics, Culture and Society, 22/2, p. 44-55.

_, 2006, Mon histoire des femmes, Paris, Seuil (coll. « Points Seuil », 2008).

—, 2009, Histoire de chambres, Paris, Seuil.

Schmitt Pantel Pauline (dir.), 1991, L'Antiquité, volume 1 de Georges Duby \& Michelle Perrot (dir.), Histoire des femmes en Occident, 5 vols, Paris, Plon, 19911992 (réédition, Paris, Perrin, coll. «Tempus », 2002).

ScOTT Joan Wallach, 1988, Gender and the Politics of History, New York, Columbia, University Press.

—, 1991, « La travailleuse », in Geneviève FraIsSE \& Michelle Perrot (dir.), Le XIXe siècle, volume 4 de Georges Duby \& Michelle Perrot (dir.), Histoire des femmes en Occident, op. cit., p. 419-444.

SCOTT Joan Wallach \& Louise TiLly, 1978, Women, Work and Family, Holt, Rinehart and Winston, 1978 (trad. fr. par Monique Lebailly : Les femmes, le travail et la famille, Marseille, Rivages, 1987).

Wieviorka Annette, 2010, Maurice et Jeannette. Biographie du couple Thorez, Paris, Fayard. 